\title{
SEMI-INCLUSIVE TAU DECAYS INVOLVING THE VECTOR OR AXIAL-VECTOR HADRONIC CURRENTS
}

\author{
S. Narison* \\ Laboratoire de Physique Mathématique (URA 768), \\ USTL, F-34060 Montpellier Cedex, France, and \\ Institut für Theoretische Physik, Universität Heidelberg, \\ Philosophenweg 16, D-6900 Heidelberg, Germany \\ and \\ A. $\operatorname{Pich}^{\dagger}$ \\ CERN, CH-1211 Geneva 23, Switzerland
}

\begin{abstract}
The theoretical QCD predictions for the semi-inclusive hadronic $\tau$ decays into an even/odd number of pions (and kaons) are updated, and compared with the corresponding sums of exclusive $\tau$-decay modes. The value of $\alpha_{s}\left(M_{\tau}^{2}\right)$ obtained from these semi-inclusive widths agrees with the one obtained from the total inclusive hadronic width. The experimental $e^{+} e^{-} \rightarrow$ Hadrons data are also used to perform additional tests in the vector sector. Using the $e^{+} e^{-}$data and varying the value of $M_{\tau}$, we show that there is a good agreement among the results obtained at different mass scales.
\end{abstract}

CERN-TH.6769/93

$\mathrm{PM} / 93-01$

HD-THEP-93-01

January 1993

\footnotetext{
* Alexander Von Humboldt Senior Fellow

${ }^{\dagger}$ On leave of absence from Departament de Física Teòrica, Universitat de València, and IFIC, Centre Mixte Universitat de València - CSIC, E-46100 Burjassot (València), Spain
} 
The total $\tau$ hadronic width has been shown $[1-10]$ to be a very good observable for testing QCD. Its inclusive nature makes it possible to perform an accurate theoretical calculation, using analyticity and the operator product expansion. Moreover, nonperturbative contributions can be shown to be strongly suppressed. The result, which is known to order $\alpha_{s}\left(M_{\tau}^{2}\right)^{3}$, turns out to be very sensitive to the value of the strong coupling [2]. Thus, the experimental measurement of this observable can be used to infer a value of $\alpha_{s}\left(M_{\tau}^{2}\right)$.

A detailed study of the $\tau$ hadronic width has already been done in ref. [6]. The (dominant) perturbative contribution has been further analyzed in ref. [7], where a resummation of known higher-order corrections has been performed; this improvement results in smaller renormalization-scheme dependence and better-defined higher-order uncertainties. We refer to those references for details and notations. The possibility of using the invariant-mass distribution of the final hadrons in $\tau$ decay to improve our control of the (small) non-perturbative contributions has been discussed in refs. [8,9]; it has been shown there that a combined fit of certain weighted integrals of the hadronic spectrum, allows us to measure simultaneously $\alpha_{s}\left(M_{\tau}^{2}\right)$ and the parameters characterizing the non-perturbative dynamics.

Predictions can also be made for those semi-inclusive $\tau$-decay widths associated with specific quark currents. As shown in refs. [2,6], one can separately compute the vector and axial-vector components of the $\tau$ hadronic width, and resolve these further into non-strange and strange contributions. The non-perturbative contributions to these semi-inclusive components are larger than for the total width; nevertheless, the theoretical accuracy is good enough to perform a meaningful QCD test. The purpose of the present letter is to provide a phenomenological study of these semi-inclusive widths, confronting the theoretical predictions with the present experimental data.

Following ref. [6], we normalize the hadronic $\tau$ decay width to the electronic one, i.e. we define the ratio

$$
R_{\tau} \equiv \frac{\Gamma\left(\tau^{-} \rightarrow \nu_{\tau} \text { hadrons }(\gamma)\right)}{\Gamma\left(\tau^{-} \rightarrow \nu_{\tau} e^{-} \bar{\nu}_{e}(\gamma)\right)}
$$

where $(\gamma)$ represents possible additional photons or lepton pairs. We will decompose the different contributions to $R_{\tau}$ into three categories:

$$
R_{\tau}=R_{\tau, V}+R_{\tau, A}+R_{\tau, S}
$$

Here $R_{\tau, V}$ and $R_{\tau, A}$ denote the vector and axial-vector contributions in the Cabibboallowed sector; $R_{\tau, S}$ contains the remaining Cabibbo-suppressed contributions.

$R_{\tau}$ can be written as a contour integral in the complex $s$-plane, running counterclockwise around the circle $|s|=M_{\tau}^{2}$,

$$
R_{\tau}=6 \pi i \oint_{|s|=M_{\tau}^{2}} \frac{d s}{M_{\tau}^{2}}\left(1-\frac{s}{M_{\tau}^{2}}\right)^{2}\left[\left(1+2 \frac{s}{M_{\tau}^{2}}\right) \Pi^{(1)}(s)+\Pi^{(0)}(s)\right] \text {. }
$$

The dynamical information is contained in the two-point correlation functions $\Pi_{i j, V / A}^{(J)}\left(q^{2}\right)$ for the vector $V_{i j}^{\mu}=\bar{\psi}_{j} \gamma^{\mu} \psi_{i}$ and axial vector $A_{i j}^{\mu}=\bar{\psi}_{j} \gamma^{\mu} \gamma_{5} \psi_{i}$ colour singlet quark 
currents $(i, j=u, d, s ; J=0,1)$. The appropriate combinations of correlators are

$$
\Pi^{(J)}(s) \equiv\left|V_{u d}\right|^{2}\left(\Pi_{u d, V}^{(J)}(s)+\Pi_{u d, A}^{(J)}(s)\right)+\left|V_{u s}\right|^{2}\left(\Pi_{u s, V}^{(J)}(s)+\Pi_{u s, A}^{(J)}(s)\right) .
$$

The contributions coming from the first two terms correspond to $R_{\tau, V}$ and $R_{\tau, A}$ respectively, while $R_{\tau, S}$ contains the contributions from the remaining terms.

The QCD predictions $[6,7,10]$ for $R_{\tau, V}, R_{\tau, A}, R_{\tau, S}$ and $R_{\tau}$ are given in Table 1 , as functions of the coupling constant $\alpha_{s}\left(M_{\tau}^{2}\right)$. The differences (within errors) with the values quoted in ref. [6] (Table 4) are due to the resummation of higher-order perturbative contributions, performed in ref. [7]. This resummation leads to a more convergent perturbative expansion and strongly reduces the renormalization scale (and scheme) dependence of the results [7]. The resummation effect is specially important at large values of $\alpha_{s}\left(M_{\tau}^{2}\right)$, where the original non-resummed expansion is non-convergent. The perturbative uncertainty, due to the unknown higher-order contributions, has been estimated by assuming an algebraic growth of the coefficients $K_{n}$, governing the perturbative expansion of the correlation function

$$
D(s) \equiv-s \frac{d}{d s} \Pi(s)=\frac{1}{4 \pi^{2}} \sum_{n=0} K_{n}\left(\frac{\alpha_{s}(s)}{\pi}\right)^{n} .
$$

In order to be conservative, and to account for all possible sources of perturbative uncertainties, we have further increased this estimate by a factor of two, i.e. we have taken $K_{4} \approx 2 K_{3}\left(K_{3} / K_{2}\right) \approx 50$ for estimating the error due to the unknown $O\left(\alpha_{s}^{4}\right)$ correction.

The experimental value for $R_{\tau}$ is actually determined by measuring the leptonic branching fractions:

$$
R_{\tau}^{B} \equiv \frac{1-B_{e}-B_{\mu}}{B_{e}}
$$

where $B_{\ell}=\Gamma\left(\tau^{-} \rightarrow \nu_{\tau} \ell^{-} \bar{\nu}_{\ell}(\gamma)\right) / \Gamma_{\tau}$ and $\Gamma_{\tau}$ is the total decay rate. An independent determination of $R_{\tau}$ can be obtained by measuring the lifetime $1 / \Gamma_{\tau}$ :

$$
R_{\tau}^{\Gamma} \equiv \frac{\Gamma_{\tau}-\Gamma_{\tau \rightarrow e}-\Gamma_{\tau \rightarrow \mu}}{\Gamma_{\tau \rightarrow e}},
$$

where $\Gamma_{\tau \rightarrow \ell}=\Gamma\left(\tau^{-} \rightarrow \nu_{\tau} \ell^{-} \bar{\nu}_{\ell}(\gamma)\right)$. Because the decays $\tau^{-} \rightarrow \nu_{\tau} \ell^{-} \bar{\nu}_{\ell}(\gamma)$ are purely electroweak processes, their rates can be calculated theoretically with great accuracy. The only unknown in eq. (7) is therefore the total decay rate $\Gamma_{\tau}$. The present results [10] for these two independent determinations of $R_{\tau}$ are

$$
\begin{aligned}
R_{\tau}^{B} & =3.64 \pm 0.03 \\
R_{\tau}^{\Gamma} & =3.55 \pm 0.06 .
\end{aligned}
$$

Although $R_{\tau}^{\Gamma}$ is slightly smaller than $R_{\tau}^{B}$, the present discrepancy between the two determinations is at the $1 \sigma$ level only ${ }^{\ddagger}$. Comparing these numbers with the predictions in Table 1 , one gets $\alpha_{s}\left(M_{\tau}^{2}\right)=0.37 \pm 0.03$ from $R_{\tau}^{B}$, and $\alpha_{s}\left(M_{\tau}^{2}\right)=0.33 \pm 0.04$ from $R_{\tau}^{\Gamma}$. The average of the two experimental determinations of $R_{\tau}$,

$$
R_{\tau}=3.62 \pm 0.03
$$

\footnotetext{
${ }^{\ddagger}$ The previous discrepancy of about $2 \sigma$ has been reduced by the small shifts on the $M_{\tau}$ and $\tau_{\tau}$ values reported recently [11].
} 
Table 1: QCD predictions $[6,7,10]$ for the different components of the $\tau$ hadronic width

\begin{tabular}{|c|ccc|c|}
\hline$\alpha_{s}\left(M_{\tau}^{2}\right)$ & $R_{\tau, V}$ & $R_{\tau, A}$ & $R_{\tau, S}$ & $R_{\tau}$ \\
& & & & \\
\hline & & & & \\
0.16 & $1.59 \pm 0.02$ & $1.49 \pm 0.03$ & $0.145 \pm 0.004$ & $3.23 \pm 0.01$ \\
0.18 & $1.61 \pm 0.02$ & $1.51 \pm 0.03$ & $0.145 \pm 0.004$ & $3.26 \pm 0.01$ \\
0.20 & $1.62 \pm 0.02$ & $1.53 \pm 0.03$ & $0.145 \pm 0.005$ & $3.29 \pm 0.01$ \\
0.22 & $1.64 \pm 0.02$ & $1.54 \pm 0.03$ & $0.145 \pm 0.005$ & $3.33 \pm 0.02$ \\
0.24 & $1.66 \pm 0.02$ & $1.56 \pm 0.03$ & $0.145 \pm 0.005$ & $3.37 \pm 0.02$ \\
0.26 & $1.68 \pm 0.02$ & $1.58 \pm 0.03$ & $0.145 \pm 0.005$ & $3.41 \pm 0.02$ \\
0.28 & $1.70 \pm 0.02$ & $1.61 \pm 0.03$ & $0.145 \pm 0.005$ & $3.45 \pm 0.02$ \\
0.30 & $1.72 \pm 0.02$ & $1.63 \pm 0.03$ & $0.145 \pm 0.006$ & $3.50 \pm 0.02$ \\
0.32 & $1.75 \pm 0.02$ & $1.65 \pm 0.03$ & $0.145 \pm 0.006$ & $3.54 \pm 0.03$ \\
0.34 & $1.77 \pm 0.02$ & $1.67 \pm 0.03$ & $0.145 \pm 0.006$ & $3.58 \pm 0.03$ \\
0.36 & $1.79 \pm 0.02$ & $1.69 \pm 0.03$ & $0.144 \pm 0.006$ & $3.63 \pm 0.03$ \\
0.38 & $1.81 \pm 0.03$ & $1.71 \pm 0.03$ & $0.144 \pm 0.007$ & $3.67 \pm 0.04$ \\
0.40 & $1.83 \pm 0.03$ & $1.73 \pm 0.03$ & $0.143 \pm 0.007$ & $3.71 \pm 0.04$ \\
0.42 & $1.85 \pm 0.03$ & $1.75 \pm 0.04$ & $0.143 \pm 0.007$ & $3.75 \pm 0.04$ \\
0.44 & $1.87 \pm 0.03$ & $1.77 \pm 0.04$ & $0.142 \pm 0.008$ & $3.79 \pm 0.04$ \\
& & & & \\
\hline
\end{tabular}

corresponds to

$$
\alpha_{s}\left(M_{\tau}^{2}\right)=0.36 \pm 0.03 .
$$

After evolution up to the scale $M_{Z}$, the strong coupling constant in Eq. (11) decreases to

$$
\alpha_{s}\left(M_{Z}^{2}\right)=0.122 \pm 0.004
$$

in amazing agreement with the present LEP average [12] (without $R_{\tau}$ ) $\alpha_{s}\left(M_{Z}^{2}\right)=$ $0.125 \pm 0.005$, and with a similar error bar. The comparison of these two determinations of $\alpha_{s}\left(\mu^{2}\right)$ in two extreme energy regimes, $M_{\tau}$ and $M_{Z}$, provides a beautiful test of the predicted running of the QCD coupling constant.

Tables 2, 3 and 4 show the contribution of the different exclusive $\tau$-decay modes to $R_{\tau, V}, R_{\tau, A}$ and $R_{\tau, S}$, respectively. We quote the results obtained from the latest compilation [13] of the Particle Data Group (PDG92), together with the modifications implied by the more recent data presented at the Ohio Workshop [11]. Although part of the latest data are still preliminary, the comparison with the official PDG92 values gives a good idea of the present data fluctuations.

The present experimental errors are still quite large ${ }^{\S}$. Moreover, the assignment of a given measurement to one of the three categories $R_{\tau, V}, R_{\tau, A}, R_{\tau, S}$ is not completely straightforward. One obviously needs to have a clean $\pi / K$ identification,

\footnotetext{
$\S_{\text {In fact, in some channels, like } 2 \pi^{-}} \pi^{+}$and $\pi^{-} 2 \pi^{0}$, the discrepancies between different experiments largely exceed the errors quoted in the world-average values [11].
} 
Table 2: Contribution of the different exclusive $\tau$-decay modes $\tau^{-} \rightarrow \nu_{\tau} V^{-}$to $R_{\tau, V}$

\begin{tabular}{|c|c|c|c|c|}
\hline \multirow{2}{*}{$\begin{array}{c}\text { Decay mode } \\
\qquad V^{-}\end{array}$} & \multicolumn{4}{|c|}{$R_{\tau \rightarrow V}$} \\
\hline & PDG92 & Ohio92 & $\begin{array}{c}e^{+} e^{-} \\
(r e f .19)\end{array}$ & $\begin{array}{c}e^{+} e^{-} \\
(\text {our estimate) }\end{array}$ \\
\hline$\pi^{-} \pi^{0}$ & $1.339 \pm 0.039$ & $1.355 \pm 0.021$ & $1.349 \pm 0.046$ & $1.346 \pm 0.040$ \\
\hline $2 \pi^{-} \pi^{+} \pi^{0}$ & $0.296 \pm 0.023$ & $0.307 \pm 0.013$ & $0.248 \pm 0.015$ & $0.268 \pm 0.040$ \\
\hline$\pi^{-3} \pi^{0}$ & $0.151 \pm 0.050$ & $0.063 \pm 0.009$ & $0.061 \pm 0.003$ & $0.057 \pm 0.010$ \\
\hline$\pi^{-} \omega$ & $0.089 \pm 0.028$ & $0.090 \pm 0.028$ & $0.128 \pm 0.018$ & $0.129 \pm 0.023$ \\
\hline $3 \pi^{-} 2 \pi^{+} \pi^{0}$ & $0.003 \pm 0.001$ & $0.003 \pm 0.001$ & - & - \\
\hline$(6 \pi)^{-}$ & - & - & $0.011 \pm 0.002$ & $0.008 \pm 0.003$ \\
\hline$\pi^{-} \pi^{0} \eta$ & $<0.062$ & $0.010 \pm 0.002$ & $0.007 \pm 0.001$ & $0.008 \pm 0.003$ \\
\hline$K^{-} K^{0}$ & $<0.015$ & $<0.015$ & $0.006 \pm 0.002$ & $0.009 \pm 0.001$ \\
\hline$\pi^{-} K^{+} K^{-}$ & $0.012 \pm 0.008$ & $0.011 \pm 0.005$ & - & $0.009 \pm 0.003$ \\
\hline$R_{\tau, V}$ & $1.821 \pm 0.094$ & $1.768 \pm 0.032$ & $1.693 \pm 0.049$ & $1.725 \pm 0.069$ \\
\hline
\end{tabular}

and to know the exact number of neutral particles (to separate the vector and axialvector contributions), which is not always the case. In order to obtain the numbers quoted in Tables 2 and 3, we have subtracted the kaon contamination, when possible. For the modes with an unknown number of neutral pions, we have assigned their contribution to the category corresponding to the minimum possible multiplicity, which is expected to be the dominant one. For instance, the PDG92 number quoted for the $\pi^{-} 3 \pi^{0}$ mode actually corresponds to $h^{-}\left(\geq 3 \pi^{0}\right)^{\top}$. Modes involving kaons present the additional problem that they can contribute (if the hadronic multiplicity is bigger than 2) both to the vector and axial-vector channels (G-parity is not a good quantum number). Although we have not made this separation in the Cabibbo-suppressed sector, we still have an ambiguity for the Cabibbo-allowed modes with an even number of kaons. We have assigned the $K K \pi$ modes to the vector sector, because they are expected [15] to be dominated by the vector amplitude. Having all these caveats in mind, the numbers shown in the tables should be taken with care, since there are additional uncertainties (hopefully small) which are probably not properly reflected in the given error bars.

In the vector channel, one can also use the information obtained, through an isospin rotation, from the isovector part of the $e^{+} e^{-}$annihilation cross-section into hadrons. The exclusive $\tau$-decay width into Cabibbo-allowed modes with $J^{P}=1^{-}$

\footnotetext{
IIn the Ohio92 case, this ambiguity has been resolved using the recent CLEO data [14] on one-prong modes with many neutrals.
} 
can be expressed as an integral over the corresponding $e^{+} e^{-}$cross-section [16-19],

$$
\begin{aligned}
R_{\tau \rightarrow V} & \equiv \frac{\Gamma\left(\tau^{-} \rightarrow \nu_{\tau} V^{-}\right)}{\Gamma_{\tau \rightarrow e}} \\
& =\frac{3 \cos ^{2} \theta_{c}}{2 \pi \alpha^{2} M_{\tau}^{8}} S_{E W} \int_{0}^{M_{\tau}^{2}} d s\left(M_{\tau}^{2}-s\right)^{2}\left(M_{\tau}^{2}+2 s\right) s \sigma_{e^{+} e^{-} \rightarrow V^{0}}^{I=1}(s),
\end{aligned}
$$

where the factor $S_{E W}=1.0194$ contains the renormalization-group improved electroweak correction at the leading logarithm approximation [20]. The results obtained in ref. [19], using the more recent update [21] of $e^{+} e^{-}$data, are given in the fourth column of Table 2. The last column of Table 2 shows our own estimate, obtained from a global fit ${ }^{* *}$ to the same $e^{+} e^{-}$data [21]. Our larger error bars reflect the data disagreements in the $4 \pi$ and $6 \pi$ channels, which we think have been underestimated in ref. [19]. The numbers quoted for the $K^{-} K^{0}$ and $\pi^{-} K^{+} K^{-}$modes have been estimated from the $\pi^{+} \pi^{-}$and $\eta \pi^{+} \pi^{-}$data respectively, assuming SU(3) symmetry and making the appropriate phase-space corrections.

Table 3: Contribution of the different exclusive $\tau$-decay modes $\tau^{-} \rightarrow \nu_{\tau} A^{-}$to $R_{\tau, A}$

\begin{tabular}{|c|c|c|}
\hline \multirow{2}{*}{ Decay mode } & \multicolumn{2}{|c|}{$R_{\tau \rightarrow A}$} \\
\cline { 2 - 3 }$A^{-}$ & PDG92 & Ohio92 \\
\cline { 2 - 3 } & & \\
\hline$\pi^{-}$ & $0.647 \pm 0.024$ & $0.637 \pm 0.020$ \\
$2 \pi^{-} \pi^{+}$ & $0.312 \pm 0.039$ & $0.461 \pm 0.017$ \\
$\pi^{-} 2 \pi^{0}$ & $0.574 \pm 0.051$ & $0.499 \pm 0.019$ \\
$3 \pi^{-} 2 \pi^{+}$ & $0.003 \pm 0.001$ & $0.003 \pm 0.001$ \\
$2 \pi^{-} \pi^{+} 2 \pi^{0}$ & - & $0.030 \pm 0.006$ \\
$\pi^{-} 4 \pi^{0}$ & - & $0.008 \pm 0.004$ \\
\hline \hline & & \\
\hline$R_{\tau, A}$ & $1.536 \pm 0.069$ & $1.638 \pm 0.033$ \\
\hline
\end{tabular}

The last row in Tables 2, 3, and 4 gives the corresponding total contribution to $R_{\tau, V}, R_{\tau, A}$ and $R_{\tau, S}$, respectively. When computing $R_{\tau, V}$, the $\pi^{-} \omega$ contribution has been weighted with the $\omega \rightarrow \pi^{0} \gamma$ branching ratio, to avoid an obvious double counting with the $2 \pi^{-} \pi^{+} \pi^{0}$ mode. Moreover, the $\pi^{-} K^{+} K^{-}$decay mode has been

\footnotetext{
"We have corrected the results of ref. [19] with the electroweak factor $S_{E W}$.

${ }^{* *}$ We use the $\pi^{+} \pi^{-}$form-factor parametrizations of refs. [18,22]. For the $\omega \pi^{0}$ mode we use the fit done in ref. [22]. The $4 \pi$ modes have been fitted with different assumptions about their resonance structure (one, two and three $\rho^{\prime}$ resonances). Due to the large data disagreements, the results in the $\pi^{+} \pi^{-} 2 \pi^{0}$ channel turn out to be very sensitive to the assumed resonance structure.
} 
Table 4: Contribution of the different exclusive $\tau$-decay modes $\tau^{-} \rightarrow \nu_{\tau} S^{-}$to $R_{\tau, S}$

\begin{tabular}{|c|c|c|}
\hline \multirow{2}{*}{ Decay mode } & \multicolumn{2}{|c|}{$R_{\tau \rightarrow S}$} \\
\cline { 2 - 3 }$S^{-}$ & PDG92 & Ohio92 \\
\hline & & \\
\hline$K^{-}$ & $0.037 \pm 0.013$ & $0.038 \pm 0.013$ \\
$K^{*-}\left(\geq 0 \pi^{0}\right)$ & $0.080 \pm 0.010$ & $0.081 \pm 0.010$ \\
$\bar{K}^{* 0} \pi^{-}\left(\geq 0 \pi^{0}\right)$ & $0.021 \pm 0.010$ & $0.021 \pm 0.010$ \\
\hline \hline$R_{\tau, S}$ & $0.138 \pm 0.019$ & $0.140 \pm 0.019$ \\
\hline
\end{tabular}

counted twice, to account for the unmeasured $\pi^{-} K^{0} \bar{K}^{0}$ contribution (isospin symmetry predicts both amplitudes to be equal). Since not all possible decay modes have been measured, the quoted sums are slight (the missing modes are suppressed ones) underestimates of the true results. This is apparent in Table 2 , where only the $3 \pi^{-} 2 \pi^{+} \pi^{0}$ part of the $(6 \pi)^{-}$mode has been taken into account in columns 2 and 3 , while the $K K \pi$ modes are missing in column 4. More important is the absence of the modes $2 \pi^{-} \pi^{+} 2 \pi^{0}$ and $\pi^{-} 4 \pi^{0}$ in the second column of Table 3 .

Table 2 shows a good agreement between the actual $\tau$-decay measurements and the numbers obtained from $e^{+} e^{-}$data. Moreover, as shown in column 3 , the $\tau$-decay data are already more accurate than the $e^{+} e^{-}$results. The "best" possible estimate of $R_{\tau, V}$ can be obtained by taking the Ohio92 results, together with the $e^{+} e^{-}$values for the $(6 \pi)^{-}$and $K^{-} K^{0}$ modes. Therefore, we quote as tentative estimates:

$$
\begin{aligned}
R_{\tau, V} & =1.78 \pm 0.03, \\
R_{\tau, A} & =1.64 \pm 0.03, \\
R_{\tau, S} & =0.14 \pm 0.02 .
\end{aligned}
$$

Adding these 3 numbers, one has the "exclusive" estimate of $R_{\tau}$ :

$$
R_{\tau}^{e x c l .}=3.56 \pm 0.05
$$

The result (17) agrees with the more direct measurements $R_{\tau}^{B}$ and $R_{\tau}^{\Gamma}$. It corresponds to

$$
\alpha_{s}\left(M_{\tau}^{2}\right)=0.33 \pm 0.04
$$

Comparing our estimates for $R_{\tau, V}$ and $R_{\tau, A}$ with the predictions in Table 1 , one gets

$$
\alpha_{s}\left(M_{\tau}^{2}\right)=0.35 \pm 0.05
$$


from the vector channel, while the axial-vector calculation implies a somewhat smaller value

$$
\alpha_{s}\left(M_{\tau}^{2}\right)=0.31 \pm 0.05
$$

Both results are in good agreement with the more precise value in eq. (11).

The experimental value in Eq. (16) for the Cabibbo-suppressed width is in excellent agreement (although errors are quite large) with the theoretical predictions in Table 1 . This is quite a non-trivial result, since the $R_{\tau, S}$ predictions are very sensitive to the power corrections. As shown in Table 1, there is practically no dependence on the value of the strong coupling in this case. In fact, the final predictions turn out to be very close to the naïve expectation $R_{\tau, S} \simeq N_{c}\left|V_{u s}\right|^{2} \simeq 0.147$, because there is a strong cancellation between the perturbative contribution and the strange-quark-mass correction. The success of the theoretical prediction could then be taken in this case as a test of the dimension- 2 contribution.

One can make an additional test in the vector channel, by using the $e^{+} e^{-}$data and varying ${ }^{\dagger}$ the value of $M \equiv M_{\tau}$ in eq. (13). The theoretical predictions for $R_{\tau, V}$ as a function of $M$ can be trivially obtained from the formulae given in refs. [6, 7 . For lower values of $M$ it is important to use the resummed perturbative expansion of ref. [7], since the bigger values of $\alpha_{s}\left(M^{2}\right)$ in that region imply that the non-resummed expansion is non-convergent. Figure 1 compares the theoretical predictions with the results obtained from $e^{+} e^{-}$data. To get the experimental points, we have used the same data fit as for obtaining the results given in the last column of Table 2. Above $2 \mathrm{GeV}$ the data is rather conflicting and incomplete, and one needs to rely on extrapolations of the fits done at lower energies; so we have not plotted this region ${ }^{\ddagger \ddagger}$. The shaded area between the two dashed curves corresponds to the theoretical prediction for $\alpha_{s}\left(M_{\tau}^{2}\right)=0.33$. The big allowed region at low values of $M$ is due to the uncertainty in the leading non-perturbative correction, which we have taken from ref. [6], i.e.

$$
\delta_{V}^{(D=6)}(M)=\delta_{V}^{(D=6)} \times\left(\frac{M_{\tau}}{M}\right)^{6}=(0.024 \pm 0.013) \times\left(\frac{M_{\tau}}{M}\right)^{6} .
$$

At the $\tau$-mass scale, $\delta_{V}^{(D=6)}\left(M_{\tau}\right)=\delta_{V}^{(D=6)}$ is a very tiny correction; however, since it scales as the sixth power of $M$, this non-perturbative contribution (and its associated uncertainty) increases very rapidly as $M$ decreases.

To show the sensitivity to the value of $\alpha_{s}\left(M_{\tau}^{2}\right)$, we have also allowed the strong coupling to change in the range $\alpha_{s}\left(M_{\tau}^{2}\right)=0.33 \pm 0.03$; one then gets the larger region between the two dot-dashed curves in Figure 1. For $M \sim M_{\tau}$, the theoretical uncertainty is dominated by the uncertainty in the input value of $\alpha_{s}\left(M_{\tau}^{2}\right)$; the error

\footnotetext{
${ }^{\dagger \dagger}$ An attempt to perform such a test, initially proposed in ref. [23], has appeared recently [24]. However, the analysis of ref. [24] is incorrect, because many important ingredients have been overlooked: electroweak and non-perturbative corrections are completely neglected (a $1 / M^{6}$ correction has been added in the revised version of this paper; we disagree, however, with the estimated numerical effect); the non-resummed perturbative expansion is used outside its validity range; not all exclusive channels have been taken into account to get the data points; moreover, the experimental error bars are ignored.

${ }_{+}$Although we found also quite good agreement between theory and experiment above $2 \mathrm{GeV}$, the big error bars of the "experimental" points make the comparison quite meaningless.
} 


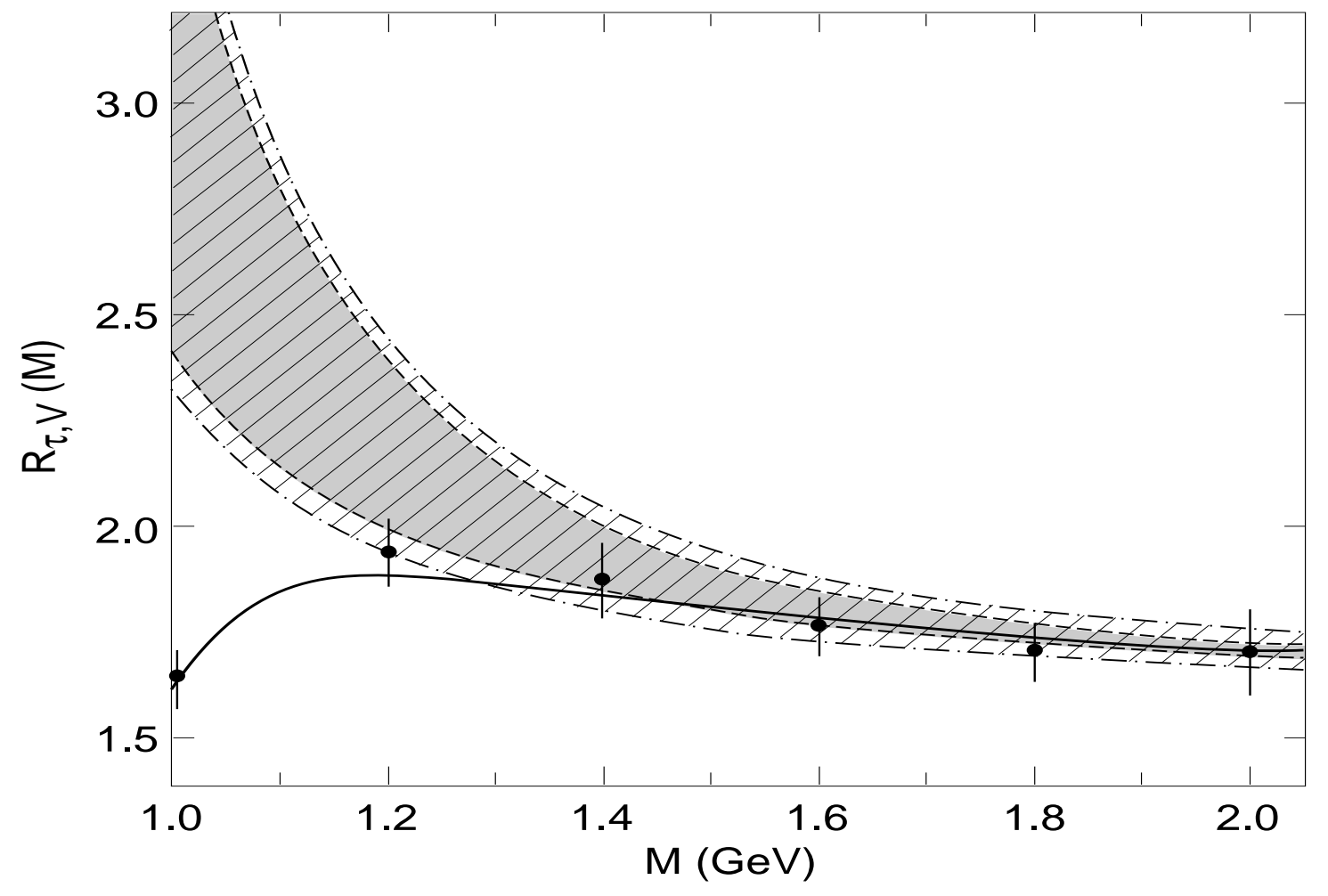

Figure 1: $R_{\tau, V}$ as a function of $M \equiv M_{\tau}$.

on $\delta_{V}^{(D=6)}$ becomes dominant for $M<1.6 \mathrm{GeV}$, and overwhelms the result for $M<$ $1.2 \mathrm{GeV}$.

One can notice that there is a good agreement between the QCD predictions and the $e^{+} e^{-}$data points for $M \geq 1.2 \mathrm{GeV}$. This confirms the role of the threshold factor $\left(1-s / M^{2}\right)^{2}$, which minimizes the theoretical uncertainties near the physical cut, and further supports the reliability of the theoretical framework used to analyze the $\tau$ hadronic width.

The departure of the theoretical prediction from the data points below $1.2 \mathrm{GeV}$ signals the important role of higher-order power corrections in this region. The subleading dimension- 8 correction has been neglected before because, at the $\tau$-mass scale, its contribution is expected to be smaller than the uncertainty on $\delta_{V}^{(D=6)}$. However, when going to smaller values of $M$, the $\delta_{V}^{(D=8)}(M)$ contribution increases much faster than the dimension- 6 one and at some point would even become dominant, indicating a breakdown of the expansion in powers of $1 / M$. We can use the lower-mass data points to make an estimate of the size of this contribution. Taking $\alpha_{s}\left(M_{\tau}^{2}\right)=0.33$ and $\delta_{V}^{(D=6)}=0.024$, a quite reasonable fit is obtained for $\delta_{V}^{(D=8)}=-0.0095$. This is shown by the continuous curve in Figure 1 . Although the $\delta_{V}^{(D=8)}(M)$ correction is tiny at $M=M_{\tau}$, its effect changes completely the predicted behaviour below $1.2 \mathrm{GeV}$. Note, however, that for this value of $\delta_{V}^{(D=8)}$ one has $\delta_{V}^{(D=8)}(M) / \delta_{V}^{(D=6)}(M)=-1.25$ at $M=1 \mathrm{GeV}$, which puts some doubts on the applicability of the inverse power expansion at such a low scale. If one takes only into account the region above $1.2 \mathrm{GeV}$, the size of the experimental error bars 
does not allow us to make a clear statement about the size of $\delta_{V}^{(D=8)}\left(\delta_{V}^{(D=8)}=0\right.$ is compatible with the data), although smaller values of $\delta_{V}^{(D=8)}$ seem to be preferred.

To summarize, the phenomenological analysis of the semi-inclusive $\tau$-decay widths, associated with the vector and axial-vector currents, shows a very consistent pattern and gives further support to the more precise study of the total $\tau$ hadronic width. Good agreement (within the present experimental errors) is found between the theoretical predictions and the exclusive experimental data, both for the $\tau$-decay measurements and for the $e^{+} e^{-} \rightarrow$ Hadrons data. Moreover, the existing $e^{+} e^{-}$data allow us to check the stability of the resulting value of $\alpha_{s}\left(M_{\tau}^{2}\right)$, obtained at different mass scales, as explicitly demonstrated in Figure 1. Complementary analyses can be done, both for the vector and axial-vector channels, by using the invariant-mass distribution of the final hadrons in $\tau$ decay $[8,9]$. At present, the quality of these exclusive analyses is limited by the poor accuracy of the experimental data, but this should be easily improved at future tau factories and low-energy $e^{+} e^{-}$machines.

Acknowledgements: This work has been supported in part by CICYT (Spain) under grant No. AEN90-0040.

\section{References}

[1] E. Braaten, Phys. Rev. Lett. 60 (1988) 1606; Phys. Rev. D39 (1989) 1458.

[2] S. Narison and A. Pich, Phys. Lett. B211 (1988) 183.

[3] A. Pich, QCD Tests From Tau Decay Data, in Proc. Tau-Charm Factory Workshop, SLAC, 1989, ed. L.V. Beers, SLAC-Report-343 (1989), p. 416.

[4] A. Pich, Hadronic Tau-Decays and QCD, in Proc. Workshop on Tau Lepton Physics, Orsay, 1990, eds. M. Davier and B. Jean-Marie (Ed. Frontières, Gifsur-Yvette, 1991), p. 321.

[5] A. Pich, Tau Physics, CERN-TH.6237/91 (to appear in Heavy Flavours, eds. A.J. Buras and M. Lindner, Advanced Series on Directions in High Energy Physics, World Scientific, Singapore, 1992).

[6] E. Braaten, S. Narison and A. Pich, Nucl. Phys. B373 (1992) 581.

[7] F. Le Diberder and A. Pich, Phys. Lett. B286 (1992) 147.

[8] F. Le Diberder and A. Pich, Phys. Lett. B289 (1992) 165.

[9] F. Le Diberder, Measurement of the Spectral Functions and Determination of the Strong Coupling Constant, contribution to the $2^{\text {nd }}$ Workshop on Tau Lepton Physics, Columbus, Ohio, September 1992. 
[10] A. Pich, QCD Predictions for the Tau Hadronic Width and Determination of $\alpha_{s}\left(M_{\tau}^{2}\right)$, CERN-TH.6738/92 (to appear in Proc. Second Workshop on Tau Lepton Physics, Columbus, Ohio, September 1992).

[11] M. Davier, Current Issues in $\tau$-Lepton Physics, Summary talk at the $2^{\text {nd }}$ Workshop on Tau Lepton Physics, Columbus, Ohio, September 1992 [Orsay preprint LAL 92-74].

[12] K. Mönig, Proc. XXVI Int. Conf. on High Energy Physics (Dallas, 1992); S. Bethke, Proc. XXVI Int. Conf. on High Energy Physics (Dallas, 1992).

[13] M. Aguilar-Benítez et al., "Review of Particle Properties", Phys. Rev. D45, Part 2 (1992).

[14] J. Urheim, contribution to the $2^{\text {nd }}$ Workshop on Tau Lepton Physics, Columbus, Ohio, September 1992;

K.K. Gan, Proc. XXVI Int. Conf. on High Energy Physics (Dallas, 1992).

[15] J.J. Gómez-Cadenas, C.M. González-García and A. Pich, Phys. Rev. D42 (1990) 3093.

[16] Y.S. Tsai, Phys. Rev. D4 (1971) 2821.

H.B. Thacker and J.J. Sakurai, Phys. Lett. 36B (1971) 103.

[17] F.J. Gilman and S.H. Rhie, Phys. Rev. D31 (1985) 1066.

F.J. Gilman and D.H. Miller, Phys. Rev. D17 (1978) 1846.

F.J. Gilman, Phys. Rev. D35 (1987) 3541.

[18] J.H. Kühn and A. Santamaría, Z.Phys. C48 (1990) 445.

[19] S.I. Eidelman and V.N. Ivanchenko, Phys. Lett. B257 (1991) 437.

[20] W.J. Marciano and A. Sirlin, Phys. Rev. Lett. 61 (1988) 1815.

[21] S.I. Dolinsky et al., Phys. Rep. 202 (1991) 99, and references therein;

G. Cosme et al., Nucl. Phys. B152 (1979) 215;

G. Bacci et al., Nucl. Phys. B184 (1981) 31;

A. Antonelli et al., Phys. Lett. B212 (1988) 133.

[22] M.B. Causse and G. Mennessier, Z. Phys. C47 (1990) 611.

[23] J.Pumplin, Phys. Rev. Lett. 63 (1989) 576; Phys. Rev. D41 (1990) 900.

[24] T.N. Truong, Ecole Polytechnique preprint EP-CPTh.A172.0492 (unpublished) [revised version: EP-CPTh.A210.1292]. 